\title{
RECURRENT MALIGNANT APOCRINE SWEAT GLAND CARCINOMA - A CASE REPORT
}

\author{
AKHILA JANGA, MOHAMMED ZIAUDDIN, RAVI CHANDER PATLOLLA
}

Department of Clinical Pharmacy, Omega Hospitals, Karimnagar, Telangana, India. Email: akhila2795@gmail.com

Received: 30 December 2020, Revised and Accepted: 11 February 2020

\begin{abstract}
Apocrine carcinomas arise from normal or modified sweat glands. The slow-growing nature and rarity of sweat gland carcinomas complicate their diagnosis at the early stage. The incidence of PASGC is quite low at 0.0049-0.0173 cases/100,000 persons/year. A 21-year-old male patient was presented in February 2016 with small solitary mass in the right axilla with mild tenderness and pain. Underwent wide local excision and axillary dissection at our institution followed by adjuvant radio and chemotherapy. Later on, after 18 months developed local recurrence and was planned for re-surgery and a second phase of palliative radiotherapy. After a year, presented to the hospital with pin sized irregular swelling in the axilla. Sweat gland carcinomas which are rarely diagnosed create a pathological dilemma due to their unpredicted biological behavior. However, wide local excision with or without nodal dissection is the primary treatment till date. In this particular case even though patient had underwent axillary node dissection, patient had recurrence twice with no distant metastasis which is a key point to be noted. Thorough study of the available apocrine carcinoma case reports or case series, the better knowledge regarding the apocrine carcinoma can be obtained.
\end{abstract}

Keywords: Apocrine carcinoma, Recurrent, Induration, Epithelial neoplasm, Perinodal extension, Malignant.

(C) 2021 The Authors. Published by Innovare Academic Sciences Pvt Ltd. This is an open access article under the CC BY license (http://creativecommons.org/ licenses/by/4.0/) DOI: http://dx.doi.org/10.22159/ajpcr.2021v14i4.40777. Journal homepage: https://innovareacademics.in/journals/index.php/ajpcr

\section{INTRODUCTION}

Apocrine carcinoma usually appears as a single, small nodule or a multinodular mass which is characterized by symptomatic progression. Among all the tumor specimens resected surgically, sweat gland carcinoma is found to be very rare with an incidence of $<0.005 \%$ [1]. There was no proper documentation regarding the first reported case of sweat gland carcinoma. According to article by Urso, the first commonly accepted case was reported by Cornil [2]. Either due to limited knowledge or due to its indolent nature, sweat gland carcinomas' are often misdiagnosed or delayed. According to the literature review, the recommended treatment for most of the sweat gland carcinomas is the wide local excision, irrespective of the positive lymph nodes. The main objective of this case report is to provide better knowledge regarding recurrent carcinomas with malignancy.

\section{CASE DETAILS}

A 21-year-old male patient was admitted on February 3, 2016, with complaints of mild pain in the right axilla for 3 months. On examination, observed small solitary mass in the right axilla with mild tenderness (Fig. 1). Found nothing significant with bilateral breast examination and skin examination. Ultrasound examination revealed no significant lesions in either breast. There was no family history of malignancy. Fine needle aspiration cytology (FNAC) of the lesion was positive and found to show Adnexal adenocarcinoma. After all the preoperative procedures, as a radical treatment, patient was planned for surgery, Wide local excision (WLE) and right axillary clearance were done as the axillary nodes are enlarged. The post-operative histopathology report (HPE) suggestive of "Sweat Gland Carcinoma" and specimen indicates 2 out of 4 nodes dissected show metastatic tumor deposits with perinodal extension $(2 / 4+)$ and the separate axillary dissection shows 2 out of 9 nodes positive for metastatic tumor deposits (2/9+) (Fig. 2). The patient underwent adjuvant radiotherapy to the right axilla with a dose of 50Gy in 25 fractions over a period of 5 weeks. Radiation therapy was delivered using conventional technique. A month later he received 5 cycles of chemotherapy with doxorubicin $78 \mathrm{mg}$, cisplatin $117 \mathrm{mg}$, and 5Fluorouracil (5FU) $500 \mathrm{mg}$ along with pre and postchemotherapy medications.
After the completion of treatment, he was asymptomatic for about 18 months and later on he developed irregular small lump in the right axilla. FNAC of the lesion was done and found as recurrent malignant epithelial neoplasm and planned for re-surgery [WLE+ Reconstruction]. Post-operative findings noted as swelling adherence to latismus dorsi muscles. Post HPE report indicates nodular invasive tumor, PNI noted and mentioned as RECURRENT MALIGNANT SWEAT GLAND CARCINOMA-APOCRINE CARCINOMA (Fig. 3). Finally, he received a second string of palliative radiotherapy for a dose of 30 Grays in 10 fractions. Patient has discharged with a review confirmation after 15 days. During patient education, the benefit of continuing treatment plan was stressed and need for follow-up treatment was discussed. After a year, he presented to the hospital with pin sized irregular swelling in the axilla. He had been advised with further investigations, but patient was not on follow-up, which was a minor drawback in this case report.

\section{DISCUSSION}

Based upon their histological behavior, sweat gland carcinomas can be classified as apocrine and eccrine. However, due to overlapping features or rarity, apocrine carcinomas pose a significant challenge in diagnosis. Hence, definite diagnostic criteria are having not been established till date. Apocrine carcinoma usually appears as a single, small nodule or a multinodular mass which is characterized by symptomatic progression. The incidence of Primary Apocrine Sweat Gland Carcinoma (PASGC) is quite low at 0.0049 to 0.0173 cases $/ 100,000$ persons per year $[3,4]$. These types of carcinomas comprises tumors with varied biological behavior. Immunohistochemical panel, molecular studies, or decapitation secretion in eosinophilic epithelial cells is considered a key indicator of apocrine differentiation. The expression of receptor profile is still controversial. Majority of the apocrine carcinomas show positivity for estrogen and progesterone receptors, but rarely some case reports identify HER2 and EGFR receptor positivity, which makes option for the targeted therapies. As we know surgery is the primary treatment in any carcinoma, similarly wide surgical excision along with lymph node dissection is the primary recommended treatment. The effectiveness of radiation and chemotherapy in treating apocrine carcinoma has not been clearly established. However, according to the article by Cardoso and Calonje, radiotherapy may reduce the risk of relapse [5]. However, it depends on the large tumor size $(=5 \mathrm{~cm})$, 


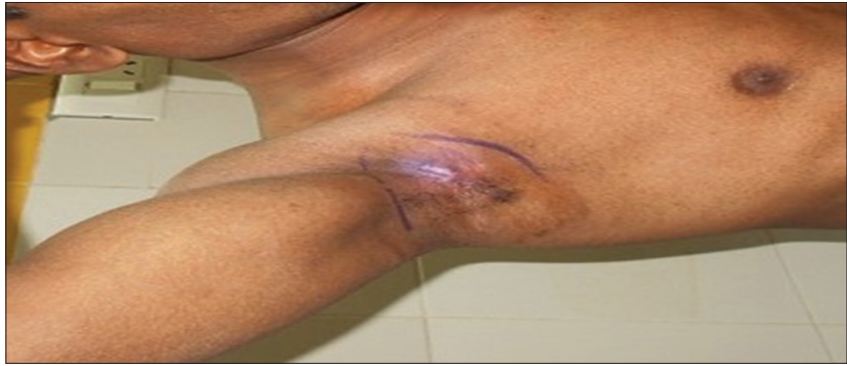

Fig. 1: Solitary swelling in the right axilla

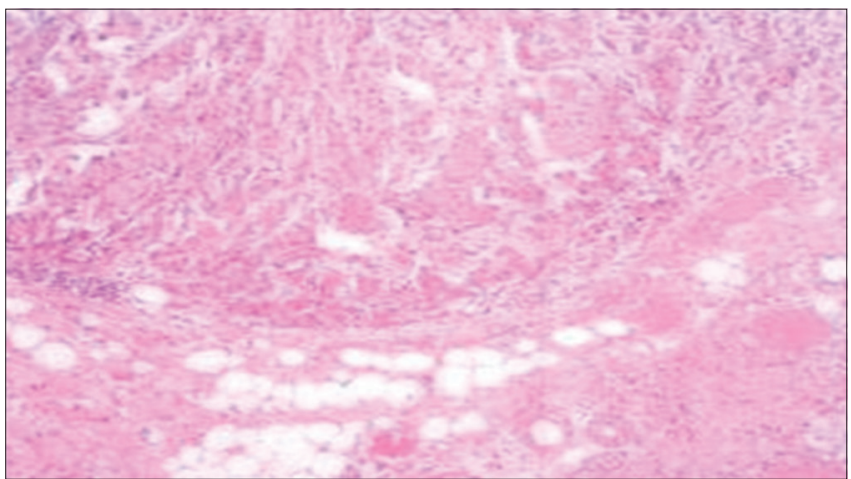

Fig. 2: Microphotograph (H\&E) section (08-02-2016)

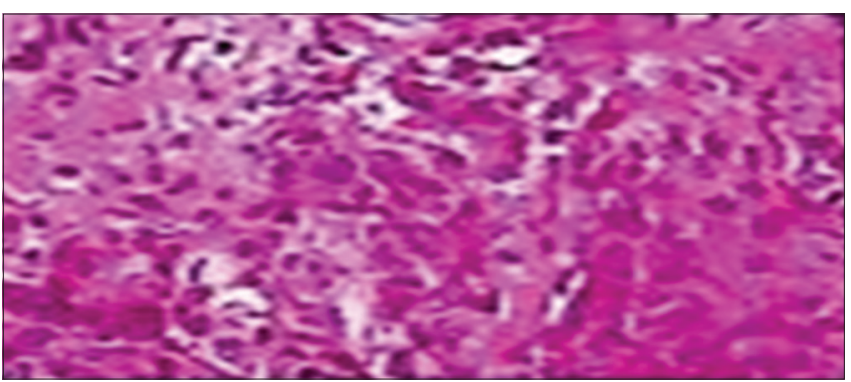

Fig. 3: Microphotograph (H\&E) section (22-08-2018)

positive resection margin, moderately to poorly differentiated tumors, or vascular or lymphatic invasion, high metastatic potential, and receptor status [3]. A 10 -year disease-free survival rate of $56 \%$ in the absence of lymph node metastasis is observed which falls to $9 \%$ if nodes are involved [6]. Vasilakaki et al., in their article, mentioned that prophylactic nodal dissection does not reduce the incidence of local recurrence [7].

\section{CONCLUSION}

Sweat gland carcinomas which are rarely diagnosed create a pathological dilemma due to their unpredicted biological behavior. Even though the effectiveness of radiotherapy and chemotherapy were not established, they were showing improved response in cases with metastasis and high-grade tumors. A prospective study should be commenced to assess the adjuvant antihormonal therapy in receptor-positive Sweat gland carcinomas. However wide surgical excision is the primary treatment till date. In this particular case even though patient had underwent axillary node dissection, patient had recurrence twice with no distant metastasis which is a key point to be noted. Due to limited number of reported cases, its a bit difficult to identify the prognostic factors and histological patterns. Through the thorough study of the available apocrine carcinoma case reports or case series, the better knowledge regarding the apocrine carcinoma can be obtained.

\section{DECLARATIONS}

\section{Ethical approval}

Not required.

\section{Consent}

Written consent was obtained from patient for publication of this case report and accompanying images.

\section{AVAILABILITY OF DATA AND MATERIALS}

The data that support the finding of this study are available from the associated institution, but restrictions apply to the availability of these data, which were used under license for the current study and are not publicly available. Data are however available from the corresponding author upon reasonable request with permission of the institution.

\section{ACKNOWLEDGMENT}

We are thankful to Dr.Rajeev Chowdary, Dr.Pradyumna Reddy, Omega Sushruta hospitals centre head for immense support.

\section{AUTHORS CONTRIBUTION}

Data collection and analysis done by Akhila Janga, literature search and review done by Mohammed Ziauddin and Ravi Chander Patlolla. Manuscript revision done by Dr. Rajiv Chowdary, Dr. Pradyumna Reddy, Dr. Abid Ali Mirza. All authors drafted and approved the final manuscript to be published.

\section{CONFLICTS OF INTREST}

The authors have no conflicts of interest.

\section{FUNDING}

No funding sources.

\section{REFERENCES}

1. Kshirsagar AY, Wader JV, Nagur B, Biradar S, Savsaviya J, Chotai T, et al. Case report: A rare case of eccrine carcinoma. Int J Surg Case Rep 2015; 15:149-51.

2. Urso C. Carcinomas of sweat glands: Report of 60 cases. Arch Pathol Lab Med 2001;125:498-505.

3. Seong MK, Kim EK, Han K, Seol H, Kim HA, Noh WC. Primary apocrine sweat gland carcinomas of the axilla: A report of two cases and a review of the literature. World J Surg Oncol 2015;13:59.

4. Gatti AP. Apocrine sweat gland adenocarcinoma: A rare case report and review. Int J Surg Case Rep 2017;36:78-81

5. Cardoso JC, Calonje E. Malignant sweat gland tumours: An update. Histopathology 2015;67:589-606.

6. Chintamani, Sharma RD, Badran R, Singhal V, Saxena S, Bansal A. Metastatic sweat gland adenocarcinoma: A clinico-pathological dilemma. World J Surg Oncol 2003;1:13.

7. Vasilakaki T, Skafida E. Primary cutaneous apocrine carcinoma of sweat glands: A rare case report. Case Rep Oncol 2011;4:597-601. 